\title{
The Prostate Cancer Intervention Versus Observation Trial:VA/ NCI/AHRO Cooperative Studies Program \#407 (PIVOT): Design and Baseline Results of a Randomized Controlled Trial Comparing Radical Prostatectomy With Watchful Waiting for Men With Clinically Localized Prostate Cancer
}

\author{
Timothy J. Wilt
}

Correspondence to: Timothy J. Wilt, MD, MPH, Minneapolis VA Center for Chronic Disease Outcomes Research, 1 Veterans Drive (111-0), Minneapolis, MN 55417 (e-mail: tim.wilt@ med.va.gov).

Prostate cancer is the most common noncutaneous malignancy and the second leading cause of cancer death in men. In the United States, $90 \%$ of men with prostate cancer are more than age 60 years, diagnosed by early detection with the prostatespecific antigen (PSA) blood test, and have disease believed confined to the prostate gland (clinically localized). Common treatments for clinically localized prostate cancer include watchful waiting (WW), surgery to remove the prostate gland (radical prostatectomy), external-beam radiation therapy and interstitial radiation therapy (brachytherapy), and androgen deprivation. Little is known about the relative effectiveness and harms of treatments because of the paucity of randomized controlled trials. The Department of Veterans Affairs/National Cancer Institute/Agency for Healthcare Research and Quality Cooperative Studies Program Study \#407:Prostate Cancer Intervention Versus Observation Trial (PIVOT), initiated in 1994, is a multicenter randomized controlled trial comparing radical prostatectomy with WW in men with clinically localized prostate cancer. We describe the study rationale, design, recruitment methods, and baseline characteristics of PIVOT enrollees. We provide comparisons with eligible men declining enrollment and men participating in another recently reported randomized trial of radical prostatectomy vs WW conducted in Scandinavia. We screened 13022 men with prostate cancer at 52 US medical centers for potential enrollment. From these, 5023 met initial age, comorbidity, and disease eligibility criteria, and a total of 731 men agreed to participate and were randomized. The mean age of enrollees was 67 years. Nearly one-third were African American. Approximately $85 \%$ reported that they were fully active. The median PSA was $7.8 \mathrm{ng} / \mathrm{mL}$ (mean $10.2 \mathrm{ng} / \mathrm{mL}$ ). In three-fourths of men, the primary reason for biopsy leading to a diagnosis of prostate cancer was a PSA elevation or rise. Using previously developed tumor risk categorizations incorporating PSA levels, Gleason histologic grade, and tumor stage, it was found that approximately $40 \%$ had low-risk, $34 \%$ had medium-risk, and $21 \%$ had high-risk prostate cancer based on local histopathology. Comparison to our national sample of eligible men declining PIVOT participation as well as to men enrolled in the Scandinavian trial indicated that PIVOT enrollees are representative of men being diagnosed and treated in the United States and quite different from men in the Scandinavian trial. PIVOT enrolled an ethnically diverse population representative of men diagnosed with prostate cancer in the United States. Results will yield important information regarding the relative effectiveness and harms of surgery compared with WW for men with predominately PSA-detected clinically localized prostate cancer.

J Natl Cancer Inst Monogr 2012;45:184-190

Treatment of early-stage prostate cancer remains controversial, especially for tumors detected with prostate specific antigen (PSA) testing. Approximately $90 \%$ of men with prostate cancer are diagnosed based on findings from early detection using the PSA blood test and have disease considered confined to the prostate gland (clinically localized disease) (1). Treatment goals are to prevent prostate cancer death and disability while minimizing intervention-related complications. Common treatments include watchful waiting $(W W)$, also referred to as expectant management, active surveillance with potential initiation of surgical or radiation treatment based on disease progression or severity from periodic digital rectal examinations, PSA tests and prostate biopsies, surgical removal of the prostate gland (radical prostatectomy, RP), externalbeam and interstitial radiotherapy (brachytherapy), and androgen deprivation (AD). Radical prostatectomy is the most common treatment for localized prostate cancer, with approximately 60 000 radical prostatectomies performed annually (2). However, three recent comprehensive systematic reviews have concluded that there is a paucity of high-quality evidence to guide patients, their families, and health-care providers regarding the comparative effectiveness and morbidity of treatments for clinically localized prostate cancer, especially in men with PSA-detected disease (3-5). Among existing randomized controlled trials (RCTs), few assessed between rather than within treatment category differences. 
Randomized trials were rarely adequately powered to assess overall or disease-specific survival or metastatic spread. The three RCTs comparing surgery with WW or radiation therapy were conducted before prostate cancer detection with PSA testing (6-8).

Department of Veterans Affairs/National Cancer Institute/ Agency for Healthcare Research and Quality (VA/NCI/AHRQ) Cooperative Studies Program (CSP) Study \#407, Prostate Cancer Intervention Versus Observation Trial (PIVOT), is a multicenter RCT comparing RP with WW. The primary aim of PIVOT is to compare all-cause mortality between the RP and WW groups. Secondary aims include comparison of prostate cancer mortality, progression, and disease-specific quality of life. We describe the design and baseline characteristics of enrollees. We also compare PIVOT enrollees with men evaluated for entry but not enrolled, and men enrolled in a Scandinavian RCT also comparing RP with WW (7).

\section{PIVOT Study Management}

PIVOT is primarily funded and coordinated by the VA Cooperative Study Program. Additional financial support has been provided by NCI and AHRQ. PIVOT is a multicenter RCT comparing two common treatment options for clinically localized prostate cancer (RP vs WW with delayed palliative intervention upon disease progression). Each study site has its own local principal investigator and study coordinator. Patient safety and trial outcomes are monitored at least annually by an independent multidisciplinary Data and Safety Monitoring Committee.

\section{Study Population}

Enrollment was initiated in November 1994 and completed in January 2002. Follow-up was completed in early 2010. Subjects were recruited from $44 \mathrm{VA}$ and 8 NCI sites across the United States. We did not employ any advertising or financial incentives. A total of 13022 men were entered onto study logs as potentially eligible (Figure 1). Based upon further chart review, 6707 met initial age, comorbidity, and prostate cancer-specific eligibility criteria and received detailed information about study participation. From these, 5023 were considered likely to be eligible, and a total of 731 men (14.5\% of likely eligible individuals) agreed to participate and were randomized to RP $(n=364)$ or WW $(n=367)$.

We specifically designed a large multicenter randomized trial to enroll a nationally representative group of patients that would receive study treatments by a large number of physicians across the United States. The randomization process was designed to create comparable treatment groups and to reduce the risk of imbalances across treatment groups at a particular site. Randomization was stratified by site and was implemented via a central interactive telephone system. Our primary recruitment goal was to enroll a broad spectrum of patients and tumor types (tumor stage, histological grade, and PSA levels) that would be considered candidates for RP and in whom there was clinical uncertainty regarding treatment effectiveness and harms. Because African American men are at increased risk for developing and dying from prostate cancer, a goal of recruitment was to enroll a high percentage of African American men. Eligible men had to have biopsy-proven clinically localized prostate cancer (T1-T2, NxM0) of any histologic grade, diagnosed within the past 12 months, PSA value $\leq 50 \mathrm{ng} / \mathrm{mL}$, age $\leq 75$ years, bone scan negative for metastatic disease, an estimated life expectancy of at least 10 years, and judged to be medically fit for RP.

\section{Study Design}

PIVOT (Figure 2) was designed as a pragmatic trial to reflect contemporary clinical practice using the two treatment strategies. As such, the exact type of RP (eg, retropubic, transperineal, use of lymph node dissection) was left to the discretion of the operating surgeon. Additional follow-up and interventions were also at the discretion of the participant's physician, but the type and reason were recorded. Men randomized to $W W$ were offered palliative (noncurative) therapies (eg, transurethral resection of the prostate for local progression causing urinary obstruction, $\mathrm{AD}$, and/or targeted radiation therapy for evidence of distant spread). Interventions for asymptomatic progression (eg, changes in PSA value) were discouraged. Protocol breaches in the WW group consisted of therapies performed for "curative intent," ie, RP, externalbeam radiotherapy, or brachytherapy. Scheduled study visits were every 6 months for a minimum of 8 years, maximum of 15 years, or patient death. Bone scans to assess for metastases were scheduled for years $5,10,15$, or end of study.

The primary endpoint is all-cause mortality. Secondary endpoints include prostate cancer-specific mortality. Additional outcomes are bone metastases, systemic progression, general and disease-specific quality of life, and harms of therapy including 30-day perioperative mortality and morbidity and longer-term urinary, bowel, and erectile dysfunction. A three-member committee comprising two urologists and a general internist adjudicates all deaths to determine the cause of death. Analysis will be by intention-to-treat, though a sensitivity analysis according to treatment received will also be conducted.

An original sample size calculated at 2000 was estimated to provide $90 \%$ power to detect a $15 \%$ relative reduction $(5 \%$ absolute reduction) in all-cause mortality with RP vs WW, assuming a median survival of 12 years. Recruitment difficulties limited the feasibility of achieving this sample size. We conducted revised sample size estimates based on baseline characteristics of the first 200 enrollees. We estimated that enrollment of 740 men over a 7 -year period with additional 8 years of study follow-up (range $8-15$ years) would provide $91 \%$ power to detect a $25 \%$ relative reduction in all-cause mortality at a median follow-up of 10 years. Predefined subgroups included age ( $>65$ vs $<65$ years), race, PSA level (as a continuous variable), tumor stage (T1 vs T2), Gleason histological tumor grade (Gleason score 2-6: histological evidence of low to moderate tumor aggressive vs Gleason score 7-10: intermediate to high tumor aggressive), and D'Amico tumor risk score (low, medium, high) incorporating tumor stage, histological grade, and PSA level.

\section{Laboratory and Pathologic Assessments}

Eligibility and baseline characteristics data including PSA values were determined by the local study sites. A central PSA laboratory was used to assay follow-up PSA values on enrollees. In an attempt 


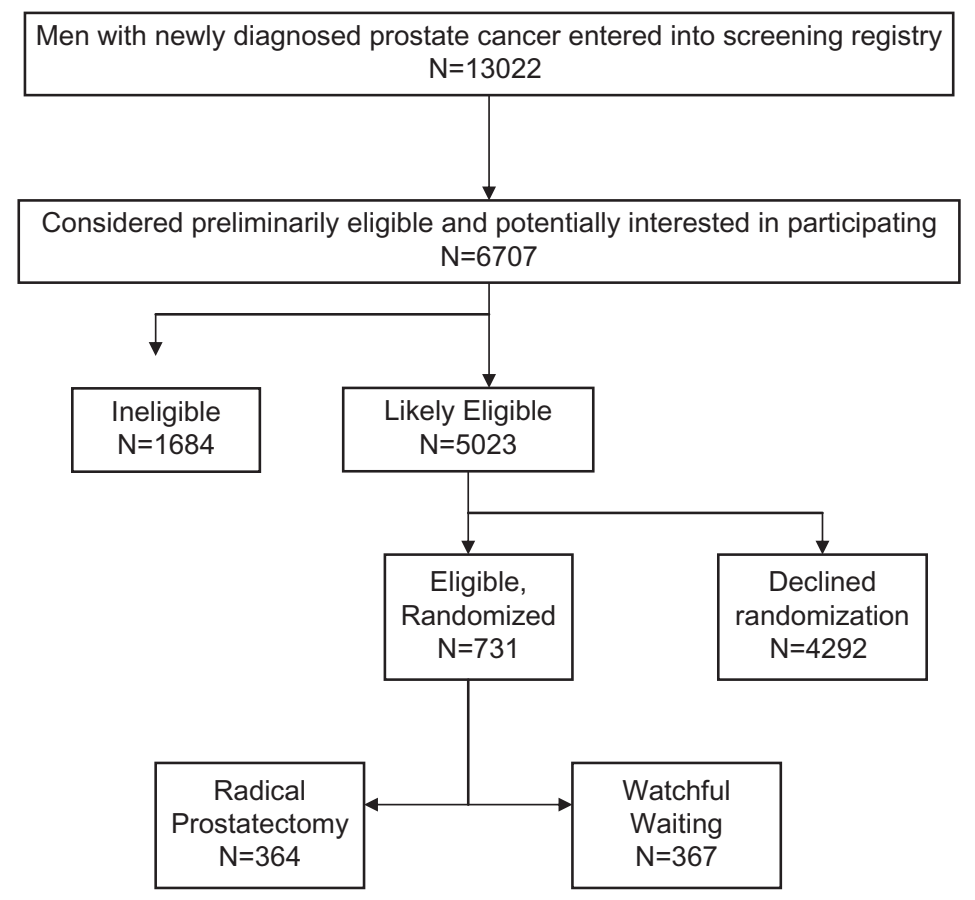

Figure 1. Participant flow diagram.

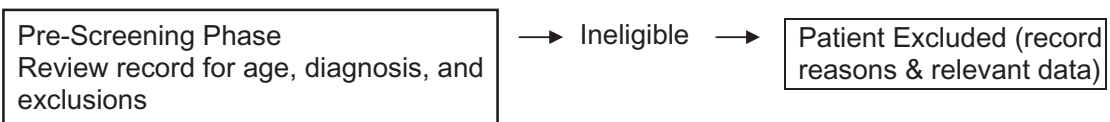

Male, age $\leq 75$, CaP present \& PSA, bone

scan meet cut point

If $\mathrm{CaP}$ but unknown lab, schedule tests

Screening Visit 1

Review videotape and written info

Brief questionnaire

Obtain laboratory data if necessary

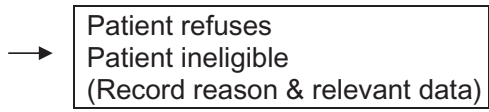

Patient ineligible
(Record reason \& relevant data)

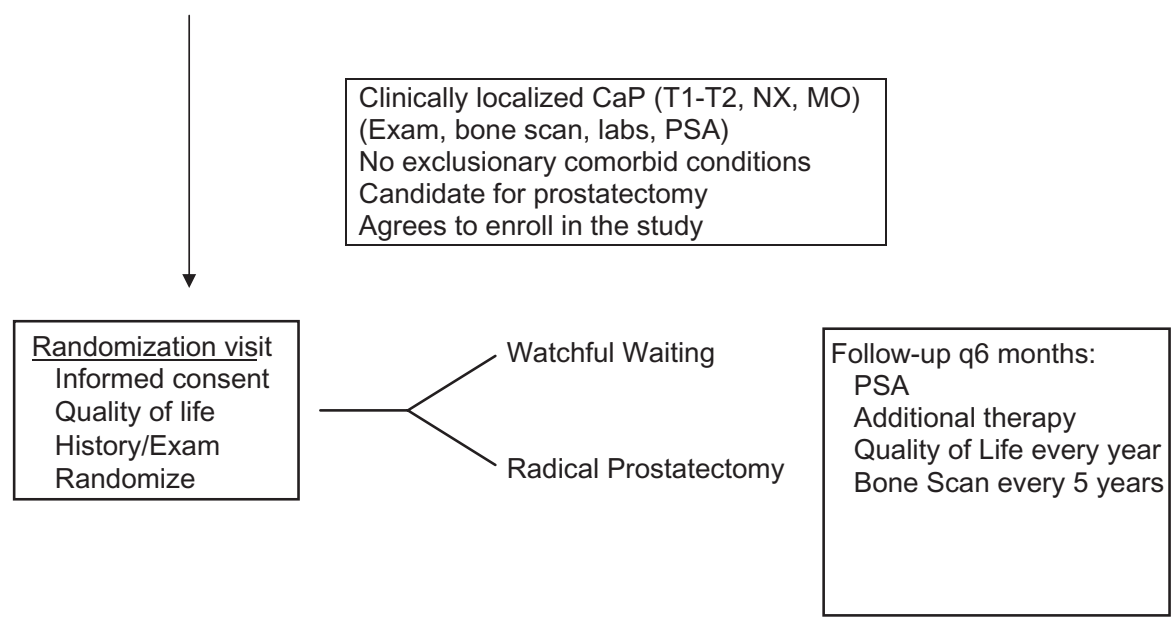

Figure 2. Prostate Cancer Intervention Versus Observation Trial (PIVOT) screening and treatment protocol. 
to reduce interventions for asymptomatic biochemical progression in the WW group, central PSA values were blinded to the investigators unless they exceeded $20 \mathrm{ng} / \mathrm{mL}$. Investigators were permitted to obtain local PSA measurements if, in their opinion, it was required to assist in the clinical diagnosis and management of the individual. A central histopathology center based at the Baylor College of Medicine, Houston, TX, reviewed diagnostic prostate biopsies and RP specimens. Central review of the diagnostic prostate biopsies was not required for study entry.

\section{Patient Characteristics}

We collected patient demographic and clinical characteristics at baseline for potentially eligible individuals. These included age, race, and self-rated health status. We also collected information on PIVOT enrollees related to marital status, education, activities of daily living, and comorbid conditions. We assessed conditions comprising the Charlson comorbidity index (CCI). The CCI encompasses 19 medical conditions and severity measures. The CCI has exhibited substantial prognostic power for both survival and treatment-related complications in numerous retrospective studies. CCI medical conditions include diabetes; congestive heart failure; coronary, cerebral, and peripheral vascular disease; chronic pulmonary disease; alcohol abuse; and dementia (9).

\section{Statistical Methods}

We compared baseline patient and tumor characteristics between the randomized treatment arms among PIVOT enrollees using $t$ tests for means and continuous variables including age, PSA mean, and Gleason score mean. Categorical variables, such as age group, marital status, and so on were compared between treatment groups using $\chi^{2}$ procedures. Similar statistical tests were used when evaluating comparability of all PIVOT enrollees as a group vs men potentially eligible but declining randomization. A $P$ value of .05 without correction was used as the statistical level of significance for these comparisons.

\section{Results}

Baseline demographic and tumor characteristics for enrollees are shown in Tables 1 and 2. There were no statistically significant differences between the randomized groups. The mean age was 67 years. Nearly one-third were black, 85\% reported that they were fully active regarding activities of daily living, and more than $80 \%$ had one or fewer Charlson comorbid conditions. The median PSA was $7.8 \mathrm{ng} / \mathrm{mL}$ (mean = 10.1). In three-fourths of men, the primary reason for biopsy leading to a diagnosis of prostate cancer was a PSA elevation or rise. One-half had stage T1c disease (not palpable, PSA-detected), and one-fourth had Gleason 7 or higher histological scores. Using tumor risk categorization, 40\% had low-risk, 34\% intermediate-risk, and 21\% high-risk prostate cancer (10). Based on central pathology review, $48 \%$ of men had Gleason 7 or higher histological scores, and $66 \%$ had intermediate- or high-risk tumor categories. Based on central pathology review, $48 \%$ had Gleason 7 or higher histological scores, and 66\% had intermediate- or high-risk tumor categories.
As noted previously, approximately $15 \%$ of potentially eligible men agreed to randomization. We compared baseline demographic, tumor, and health status characteristics of PIVOT enrollees with that of other cohorts of men with prostate cancer and with that of men eligible but declining randomization to assess external validity (Table 2). Compared with men who were PIVOT eligible but declined enrollment, PIVOT enrollees were slightly older, more likely to be African American, have well-differentiated prostate cancer, and report their health status as excellent or very good. The cohort of all screen registrants (including those not satisfying eligibility requirements) was slightly older, less likely to be African American, had higher mean PSA values, and a PSA value 20 or higher than PIVOT enrollees. The most frequent reasons for PIVOT exclusion among the cohort of all screened registrants were nonlocalized disease (15\%), life expectancy less than 10 years $(8.5 \%)$, debilitating illness (6.1\%), age greater than 75 years (4.9\%), and PSA level greater than $50 \mathrm{ng} / \mathrm{mL}(4.3 \%)$. We compared results with that in men enrolled in a similar, but smaller, ongoing RCT of RP vs WW conducted in several Scandinavian countries (SPCG-4) (7). Compared with PIVOT enrollees, men in SPCG-4 were slightly younger, had higher mean PSA values, and had many fewer cancers detected by PSA testing, and there appeared to be less racial diversity.

\section{Discussion}

The preferred management of clinically localized prostate cancer is not known, due in large part to the paucity of RCTs comparing the effectiveness and harms across primary treatment options $(3-5,7)$. In these studies, enrollees, tumor characteristics, and/ or interventions may not be representative of US men currently being diagnosed and receiving treatment in the United States. For example, the SPCG-4 trial reported that RP reduced death from all causes, prostate cancer, and distant metastases compared with WW by $6.6 \%, 6.1 \%$, and $6.7 \%$ and $11.7 \%$, respectively, at 15 years. Differences remained stable after 10 years. Other outcomes, including local recurrence and/or progression, institution of hormonal therapy, and use of other palliative therapies, also favored RP. Overall mortality was not statistically significantly different between groups but favored RP (13.5 vs $19.5 ; P=.09)$ (7). Effectiveness was limited to men younger than age 65 years but was not associated with Gleason score or baseline PSA level. However, the majority of men enrolled in the SPCG-4 study did not have PSA-detected disease. A smaller trial found no significant overall survival differences between RP and WW (risk difference, 0\% [95\% CI $=-19 \%$ to $18 \%$ ]) (6). This study was conducted before the development of the PSA blood test and may not reflect outcomes with modern surgical treatment. RP reduced disease recurrence at 5 years compared with external-beam radiation in one small trial conducted more than 25 years ago (14\% vs 39\%; risk difference, $21 \% ; P=.04)(8)$.

The only other published randomized trial of interventions for localized prostate cancer assessed outcomes within rather than between primary therapies. Results of a recent systematic review found no randomized trials comparing primary $\mathrm{AD}$ with other primary therapies such as surgery or radiation therapy (3). No randomized trials evaluated emerging technologies such as brachytherapy, cryotherapy, robotic RP, and photon-beam or 
Table 1. Baseline characteristics*

\begin{tabular}{|c|c|c|c|}
\hline & Observation ( $n=367)$ & $\operatorname{RP}(n=364)$ & $P$ value \\
\hline Mean age, y (SD) & $66.8(5.6)$ & $67.0(5.2)$ & .60 \\
\hline Age grouping (\%) & & & .61 \\
\hline $40-49$ years & $3(0.8)$ & $1(0.3)$ & \\
\hline $50-59$ years & $32(8.7)$ & $39(10.7)$ & \\
\hline $60-69$ years & $211(57.5)$ & $203(55.8)$ & \\
\hline $70-75$ years & $121(33.0)$ & $121(33.2)$ & \\
\hline Race $(\%)$ & & & .53 \\
\hline Black & $121(33.0)$ & $111(30.5)$ & \\
\hline White & $220(60.0)$ & $232(63.7)$ & \\
\hline Other & $26(7.1)$ & $21(5.8)$ & \\
\hline Activities of daily living (\%) & & & .17 \\
\hline Fully active & $310(84.5)$ & 312 (85.7) & \\
\hline Ambulatory & $52(14.2)$ & $41(11.3)$ & \\
\hline No work/self-care/confined & $5(1.4)$ & $11(3.0)$ & \\
\hline Smoking (\%) & & & .63 \\
\hline Current smoker & $106(28.9)$ & $96(26.4)$ & \\
\hline \multicolumn{4}{|l|}{ History/current (\%) } \\
\hline Myocardial infarction & $43(11.7)$ & $37(10.2)$ & .50 \\
\hline Congestive heart failure & $8(2.2)$ & $3(0.8)$ & .13 \\
\hline Peripheral vascular disease & $20(5.5)$ & $15(4.1)$ & .43 \\
\hline Cerebral vascular disease & $16(4.4)$ & $7(1.9)$ & .06 \\
\hline Stroke & $18(4.9)$ & $14(3.9)$ & .48 \\
\hline Diabetes & $59(16.1)$ & $56(15.4)$ & .80 \\
\hline COPD & $25(6.8)$ & $37(10.2)$ & .10 \\
\hline $\mathrm{CCl}$ score $(\%)$ & & & .20 \\
\hline 0 conditions & $204(55.6)$ & $206(56.6)$ & \\
\hline 1 condition & $103(28.1)$ & $108(29.7)$ & \\
\hline 2 or more conditions & $60(16.4)$ & $47(12.9)$ & \\
\hline \multicolumn{4}{|l|}{ Tumor characteristics } \\
\hline PSA (ng/ml): Mean (SD) & $10.2(7.9)$ & $10.1(7.4)$ & .89 \\
\hline Median & 7.7 & 7.9 & \\
\hline PSA categories (\%) & & & .89 \\
\hline$<4.0$ & $40(10.9)$ & $42(11.5)$ & \\
\hline $4.0-10.0$ & $201(54.8)$ & $196(53.9)$ & \\
\hline $10.1-19.9$ & $88(24.0)$ & $88(24.2)$ & \\
\hline 20.0-49.9 & $37(10.1)$ & $38(10.4)$ & \\
\hline Clinical stage (\%) & & & .38 \\
\hline TX & $1(0.3)$ & $1(0.3)$ & \\
\hline T1A (A1) & $11(3.0)$ & $4(1.1)$ & \\
\hline $\mathrm{T} 1 \mathrm{~B}(\mathrm{~A} 2)$ & $9(2.5)$ & $5(1.4)$ & \\
\hline $\mathrm{T} 1 \mathrm{C}$ & 183 (49.9) & $185(50.8)$ & \\
\hline T2A (B1) & $85(23.2)$ & $96(26.4)$ & \\
\hline T2B (B2) & $44(12.0)$ & $47(12.9)$ & \\
\hline $\mathrm{T} 2 \mathrm{C}$ & $33(9.0)$ & $24(6.6)$ & \\
\hline T3C & $0(0.0)$ & $1(0.3)$ & \\
\hline Gleason score (\%) & & & .41 \\
\hline$\leq 4$ & 75 (20.4) & $82(22.5)$ & \\
\hline $5-6$ & $186(50.7)$ & $172(47.3)$ & \\
\hline 7 & $64(17.4)$ & $69(19.0)$ & \\
\hline $8-10$ & $22(6.0)$ & $29(8.0)$ & \\
\hline Mean Gleason score (SD) & $5.7(1.4)$ & $5.6(1.4)$ & .80 \\
\hline Histologic grade $(\%) \dagger$ & & & .66 \\
\hline Well differentiated & $75(20.4)$ & $82(22.5)$ & \\
\hline Moderately well differentiated & $261(71.1)$ & $247(67.9)$ & \\
\hline Poorly differentiated & $22(6.0)$ & $29(8.0)$ & \\
\hline Unknown & $5(1.4)$ & $4(1.1)$ & \\
\hline Tumor risk category $(\%) \ddagger$ & & & .37 \\
\hline Low risk & $148(40.3)$ & $148(40.7)$ & \\
\hline Intermediate risk & $120(32.7)$ & $129(35.4)$ & \\
\hline High risk & $80(21.8)$ & $77(21.2)$ & \\
\hline
\end{tabular}

* $\mathrm{CCl}=$ Charlson comorbidity index; $\mathrm{COPD}=$ chronic obstructive pulmonary disease; PSA = prostate-specific antigen; RP = radical prostatectomy; $\mathrm{SD}=$ standard deviation. Adapted from Wilt et al. (17) with permission from Elsevier.

† Based on local site histology: Well differentiated = Gleason score <4; moderately well differentiated = Gleason score 5-7; poorly differentiated = Gleason score 8-10.

‡ Low risk = Gleason Score <6, PSA $<10$ and tumor stage T1a, T1b, T1c, or T2a; intermediate risk = PSA 10.1-20 ng/mL or Gleason score = 7 or stage T2b; high risk $=$ PSA $>20 \mathrm{ng} / \mathrm{mL}$ or Gleason score 8-10 or stage T2c. Four percent of men had insufficient information to determine tumor risk category scores. 
Table 2. Comparison of Prostate Cancer Intervention Versus Observation Trial (PIVOT) enrollees with men eligible but declining randomization, all PIVOT screenees, and enrollees from the Scandinavian Prostate Cancer Group Study \#4 (SPCG-4) trial*

\begin{tabular}{|c|c|c|c|c|c|}
\hline & PIVOT enrollees & $\begin{array}{c}\text { PIVOT-eligible but } \\
\text { declined }\end{array}$ & $\begin{array}{l}P \text { value PIVOT vs } \\
\text { eligible but declined }\end{array}$ & $\begin{array}{l}\text { PIVOT screen } \\
\text { registrants }\end{array}$ & SPCG-4 \\
\hline Number & 731 & 4286 & & 13022 & 695 \\
\hline Age, mean y & 67 & 66 & $<.001$ & 68 & 65 \\
\hline$<65, \%$ & 35 & 42 & & 32 & 46 \\
\hline Race, \% & & & 0.001 & & $\mathrm{n} / \mathrm{a}$ \\
\hline White & 62 & 72 & & 68 & \\
\hline Black & 32 & 24 & & 27 & \\
\hline Hispanic & 4 & 4 & & 3 & \\
\hline PSA, mean ng/mL & 10.1 & 11.0 & .52 & 47 & 12.9 \\
\hline$<4, \%$ & 11 & 10 & .39 & 9 & 18 \\
\hline $4-9.9, \%$ & 54 & 54 & & 45 & 37 \\
\hline $10-19.9, \%$ & 25 & 25 & & 23 & 28 \\
\hline $20-49.9, \%$ & 10 & 10 & & 13 & $18+$ \\
\hline$\geq 50, \%$ & 0 & 1 & & 10 & \\
\hline PSA-detected prostate cancer, $\%$ & 76 & $\mathrm{n} / \mathrm{a}$ & & $\mathrm{n} / \mathrm{a}$ & 5 \\
\hline Localized clinical stage, \% & 100 & 100 & & 85 & 100 \\
\hline $\mathrm{T} 1 \mathrm{C}$ & 50 & & & & 11 \\
\hline $\mathrm{T} 2$ & 45 & & & & 76 \\
\hline Histologic grade, \% & & & .001 & & \\
\hline Well differentiated & 25 & 18 & & 14 & 13 \\
\hline Moderately differentiated & 62 & 65 & & 61 & 70 \\
\hline Poorly differentiated & 7 & 13 & & 20 & 5 \\
\hline Undetermined & 6 & 4 & & 4 & 12 \\
\hline Health status self-rating, \% & & & .001 & $\mathrm{n} / \mathrm{a}$ & $\mathrm{n} / \mathrm{a}$ \\
\hline Excellent & 19 & 13 & & & \\
\hline Very good & 36 & 28 & & & \\
\hline Good & 31 & 28 & & & \\
\hline Fair & 12 & 6 & & & \\
\hline Poor & 0 & 0 & & & \\
\hline Not asked/answered & 2 & 24 & & & \\
\hline
\end{tabular}

* $\mathrm{n} / \mathrm{a}=$ data not available; PSA = prostate-specific antigen. Reproduced from Wilt et al. (17) with permission from Elsevier.

† PSA values at this level for SPCG-4 study were reported as $>20 \mathrm{ng} / \mathrm{ml}$.

intensity-modulated radiation. Results from observational studies were inadequate to assess comparative effectiveness, and data demonstrated wide and overlapping effectiveness estimates within and between treatments.

Likewise, comparing the incidence and severity of treatment harms is difficult because authors used wide variations in their definitions and assessments of severity of adverse events (3). In the SPCG-4 study, RP was associated with worse urinary leakage and sexual function but similar overall health and improved urinary obstructive symptoms to $W W$ at 4 years among approximately 400 men surviving and completing the survey (11). A population-based study assessed patient-reported treatment harms and satisfaction among male Medicare beneficiaries with clinically localized prostate cancer (Prostate Cancer Outcomes Study Number 4) $(3,12)$. Results indicated that urinary leakage occurring daily or more often was more common in men undergoing RP $(35 \%)$ than in those undergoing external-beam radiation therapy $(12 \%)$ or $\mathrm{AD}(11 \%)$. External-beam radiation therapy and $\mathrm{AD}$ were associated with a higher frequency of bowel urgency (3\% each) compared with RP $(1 \%)$. Inability to attain an erection was higher in men undergoing active intervention, especially $\mathrm{AD}(86 \%)$ or $\mathrm{RP}(58 \%)$, than in men receiving WW (33\%). Patient satisfaction with all selected treatments was high. More than $90 \%$ would make the same treatment decision again, and most were delighted or pleased with their treatment decision (12).
PIVOT was designed to address limitations in the existing evidence and evaluate the effectiveness and harms of two commonly used primary treatments in a broad spectrum of men currently being diagnosed and considered for treatment of clinically localized prostate cancer in the United States. No other trial has enrolled a racially diverse mix of men with prostate cancer primarily detected with PSA testing (the predominant mode of prostate cancer detection currently). The design of PIVOT was pragmatic to incorporate individual physician and patient treatment preferences consistent with current clinical practice of RP and WW. PIVOT will determine whether early intervention with surgery (RP) improves the length and quality of life in men with clinically localized prostate cancer compared with WW with delayed palliative care if and when there is evidence of symptomatic disease progression. The design, compared interventions, and the baseline demographic and tumor characteristics of PIVOT enrollees indicate that results will be relevant to the vast majority of men currently diagnosed with prostate cancer in the United States (1214). Study sample size and duration, use of a blinded adjudication committee, and comprehensive/standardized data collection will permit reliable assessment of effectiveness and harms according to clinically relevant patient and tumor characteristics and should greatly facilitate treatment decision making.

Additional RCTs are ongoing or being planned. These include the ProtecT study, a three-arm RCT based in the United Kingdom 
comparing RP with external-beam radiotherapy or active surveillance (15). Other RCTs have not been able to successfully recruit men including one comparing RP with brachytherapy for low-risk prostate cancer. Difficulty in recruitment of patients to cancer trials is not unique to prostate cancer (16). Understanding and reducing these barriers is crucial to successful completion of future RCTs and critical to determining the relative effectiveness and harms of treatments for clinically localized prostate cancer.

\section{References}

1. Siegel R, Naishadham D, Jemal A. Cancer statistics, 2012. CA Cancer 7 Clin. 2012;62(1):10-29.

2. Healthcare cost and utilization project (US). Agency for Healthcare Research and Quality Web site. http://www.ahrq.gov/data/hcup/. Accessed December 2006.

3. Wilt TJ, MacDonald R, Rutks I, Shamliyan TA, Taylor BC, Kane RL. Systematic review: comparative effectiveness and harms of treatments for clinically localized prostate cancer. Ann Intern Med. 2008;148(6):435-448.

4. Thompson I, Thrasher JB, Aus G, et al. Guideline for the management of clinically localized prostate cancer: 2007 update. American Urological Association Education and Research Web site: http://www.auanet.org/ content/guidelines-and-quality-care/clinical-guidelines/main-reports/ proscan07/content.pdf. Accessed October 17, 2012.

5. Graham J, Baker M, Macbeth F, Titshall V; Guideline Development Group. Diagnosis and treatment of prostate cancer: summary of NICE guidance. BM7. 2008;336(7644):610-612.

6. Iversen P, Madsen PO, Corle DK. Radical prostatectomy versus expectant treatment for early carcinoma of the prostate. Twenty-three year follow-up of a prospective randomized study. Scand 7 Urol Nephrol Suppl. 1995;172:65-72.

7. Bill-Axelson A, Holmberg L, Ruutu M, et al; SPCG-4 Investigators. Radical prostatectomy versus watchful waiting in early prostate cancer. $N$ Engl 7 Med. 2011;364(18):1708-1717.

8. Paulson DF, Lin GH, Hinshaw W, Stephani S. Radical surgery versus radiotherapy for adenocarcinoma of the prostate. 7 Urol. 1982;128(3):502-504.

9. Charlson ME, Pompei P, Ales KL, MacKenzie CR. A new method of classifying prognostic comorbidity in longitudinal studies: development and validation. 7 Chronic Dis. 1987;40(5):373-383.

10. D'Amico AV, Manola J, Loffredo M, Renshaw AA, DellaCroce A, Kantoff PW. 6-month androgen suppression plus radiation therapy vs radiation therapy alone for patients with clinically localized prostate cancer: a randomized controlled trial. FAMA. 2004;292(7):821-827.
11. Steineck G, Helgesen F, Adolfsson J, et al; Scandinavian Prostatic Cancer Group Study Number 4. Quality of life after radical prostatectomy or watchful waiting. $N$ Engl 7 Med. 2002;347(11):790-796.

12. Hoffman RM, Hunt WC, Gilliland FD, Stephenson RA, Potosky AL. Patient satisfaction with treatment decisions for clinically localized prostate carcinoma. Results from the Prostate Cancer Outcomes Study. Cancer. 2003;97(7):1653-1662.

13. Freedland SJ, Amling CL, Dorey F, et al; Shared Equal Access Regional Cancer Hospital Database Study Group. Race as an outcome predictor after radical prostatectomy: results from the Shared Equal Access Regional Cancer Hospital (SEARCH) database. Urology. 2002;60(4):670-674.

14. Greene KL, Cowan JE, Cooperberg MR, Meng MV, DuChane J, Carroll PR; Cancer of the Prostate Strategic Urologic Research Endeavor (CaPSURE) Investigators. Who is the average patient presenting with prostate cancer? Urology. 2005;66(5 suppl):76-82.

15. Donovan JL, Peters TJ, Noble S, et al; ProtecT Study Group. Who can best recruit to randomized trials? Randomized trial comparing surgeons and nurses recruiting patients to a trial of treatments for localized prostate cancer (the ProtecT study). 7 Clin Epidemiol. 2003;56(7):605-609.

16. Ford JG, Howerton MW, Lai GY, et al. Barriers to recruiting underrepresented populations to cancer clinical trials: a systematic review. Cancer. 2008;112(2):228-242.

17. Wilt TJ, Brawer MK, Barry MJ, et al. The Prostate cancer Intervention Versus Observation Trial:VA/NCI/AHRQ Cooperative Studies Program \#407 (PIVOT): design and baseline results of a randomized controlled trial comparing radical prostatectomy to watchful waiting for men with clinically localized prostate cancer. Contemp Clin Trials. 2009;30(1): $81-87$.

\section{Funding}

Department of Veterans Affairs Cooperative Studies Program, the National Cancer Institute and the Agency for Healthcare Research and Quality.

\section{Note}

A version of this article was previously published in (17). Reprinted with permission from Elsevier.

Affiliations of author: Minneapolis VA Center for Chronic Disease Outcomes Research and the University of Minnesota Department of Medicine, Minneapolis, MN. 